\title{
Polymer optical fiber tapering using chemical solvent and polishing
}

\author{
L. S. Supian ${ }^{1 *}$, Mohd Syuhaimi Ab-Rahman ${ }^{1}$, and Norhana Arsad ${ }^{1,2}$ \\ ${ }^{1}$ Department of Electrical and Electronic Engineering, Faculty of Engineering and Built Environment \\ ${ }^{2}$ Institute of Microelectronic and Nanotechnology, Faculty of Engineering and Built Environment \\ Universiti Kebangsaan Malaysia, 43000, Bangi, Selangor, Malaysia
}

\begin{abstract}
A method for developing polymer optical fiber (POF) directional coupler is introduced where the initial procedure includes using chemical solvent to remove the cladding, and bare out the core in order to align the unclad center of the fiber with other similar fiber to develop a coupler. The process is safe, simple, inexpensive and require low operation skill. The etched fiber offers improvement to the performance of various POF devices, i.e, couplers and sensors. Instead of relying only on silica or glass fiber, POF now can be used as an alternative to improve the network performance in short distance communication system. The measurement parameters laid out offer great outcomes. However, the couplers intended to be developed is yet to be realized, where deeper research and various experiments are needed in order to develop a simple but optimum performance coupler that can be used for various applications.
\end{abstract}

\section{Introduction}

Couplers are common passive optical device that functions to combine two or more wavelengths or signals into one single optical fiber. There have been several methods on tapering polymer optical fiber for short-haul communication device. The existing method to develop the coupler is fused coupler [1], one of the most popular techniques of manufacturing a coupler. The process is simple where at least two fibers are brought close in proximity at a certain length and fused at the particular area. The results will produce a coupler where the middle part of the fiber has diameter of around $\sim 1.0 \mathrm{~mm}$ $-1.2 \mathrm{~mm}$. The narrower cores increase the spread of the evanescent field in the cladding and this assist the coupling process. The drawback emerges through this method where it is quite impossible to control the spacing of the two cores that held close in proximity and the length of the coupling region accurately $[2,3]$.

In this particular study, chemical etching technique is used to fabricate a directional optical coupler. A quite similar but different technique [4] is already developed where the fibers are embedded into a solid material such as plastic and the flat surface is then polished until the cladding of the fibers is removed at 4 microns thick. A D-shape fiber results and the plastic is removed. The cladding is thinned out by etching using hydrofluoric acid. Two D-shape fibers are joint longitudinally along the flat areas using index-matching epoxy-resin. This technique shows better precision in terms of spacing between the two fibers and the coupling length than fused-taper method [5]. A technique of removing the cladding of polymethylmethacrylate (PMMA) polymer optical fiber using chemical solvent is used to create etched tapers at certain length in the middle part of the fiber. In consequence of tapering of the multimode optical fiber cladding, higher modes of the fiber are removed while some modes are redistributed. This technique is conventionally applicable to glass fiber. However, POF is becoming widely used in optical communication system and this would be a jump start for fabricating highly-potential coupler using this technique.

\section{Fabrication method/experiment}

Polymer optical fiber used in this work has diameter core of $\varnothing=980 \mu \mathrm{m}$ and diameter cladding of $\varnothing=20 \mu \mathrm{m}$ thick. Chemical solvent i.e. acetone is used in this studies in order to remove cladding layer. Due to the effectiveness of the solvent to impair and remove the cladding layer as reported by the research done by Merchant, Scully and Schmitt [2], pure $100 \%$ acetone is used in the experiment without any additional liquid or solvent involved or change the concentration of the solvent.

The first set of experiment involves a flat-base plate of $80 \mathrm{~mm}$ based $\left(\mathrm{x}_{1} \mathrm{~mm}\right) \times 25 \mathrm{~mm}$ height $\left(\mathrm{y}_{1} \mathrm{~mm}\right)$ as in Fig. 3(a). Pure solvent of $10 \mathrm{ml}$ are used and polymer fiber strand of about $30 \mathrm{~mm}$ are held stationary at both ends which the center part of the polymer fiber is dipped thoroughly inside the acetone. Time varies for each of the polymer fiber dipped inside the solvent. Trials of more than 20 secs failed as the fiber at the center section becomes susceptible to brittle stress fracture at certain time i.e. threshold time. Break will precede the brittleness once the fiber held is moved rigorously or tension is applied onto the polymer fiber in etching. One factor that contributes to the breaking of the fiber is the bending of the strand of fiber influenced by the height of the side of the plate. Here, the temperature is taken as default and not taken into consideration. After each of the etching process is done, the affected region is polished evenly in order to remove the cladding layer effectively. Fig. 1. shows fiber after etching process and the fiber is polished along the affected region. Cloudy surface is observed around the affected region.

* Corresponding author: cawa711@gmail.com 


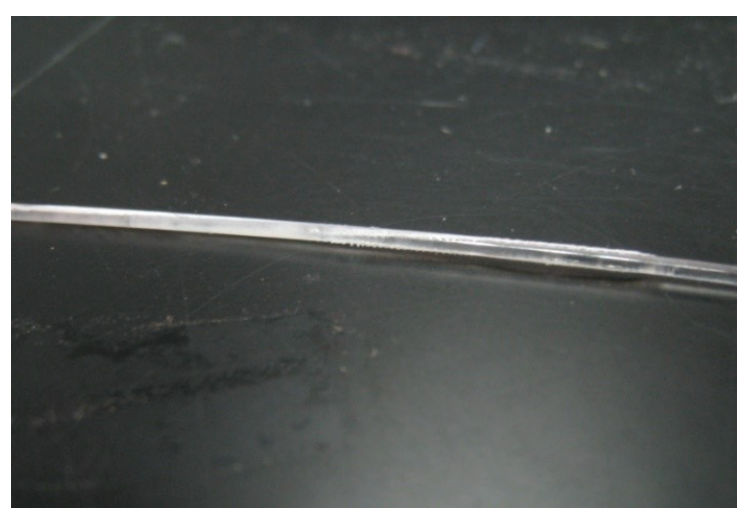

Fig. 1. Etched and polished fiber outer structure

The second set of the experiment involves different flatbase plate of $60 \mathrm{~mm}$ based $\left(\mathrm{x}_{2} \mathrm{~mm}\right) \times 10 \mathrm{~mm}$ height $\left(\mathrm{y}_{2}\right.$ $\mathrm{mm}$ )as in Fig. 3(b). Similar amount of pure solvent are used i.e. $10 \mathrm{ml}$. Due to the lower bending of the fiber strand as a factor of the lower height of the side plate, longer time are taken from initial time of 5 secs up till 35 secs for each of the polymer fiber dipped into the pure solvent. Again, the temperature is taken as default and not taken into consideration. For the second and third set of experiment, polishing is excluded. Fig. 2. shows fiber after etching process and no polishing is applied on the etched region. Less cloudy surface is observed compared to the previous polished fiber.

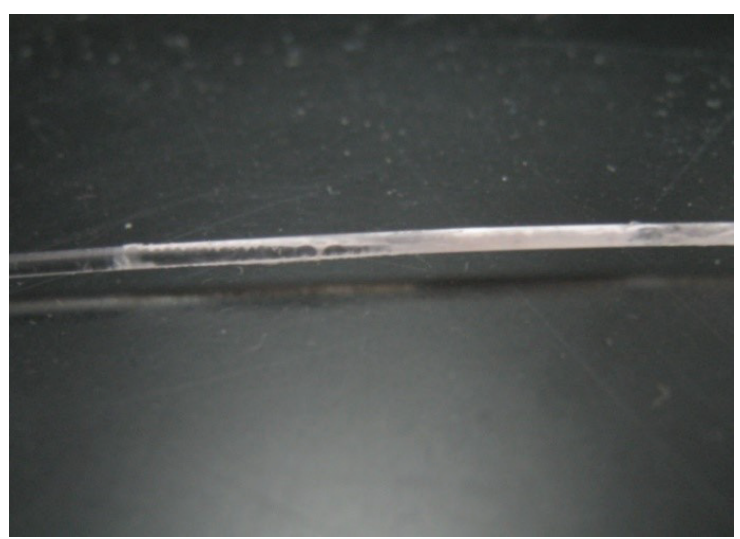

Fig. 2. Etched and unpolished fiber outer structure

After each of the experiment is completed, $1 \mathrm{ml}$ of isopropyl alcohol and $1 \mathrm{ml}$ of distilled water are used to neutralize and wash the waste of the outer cladding that have been etched before any measurement is taken.

\section{Results and analysis}

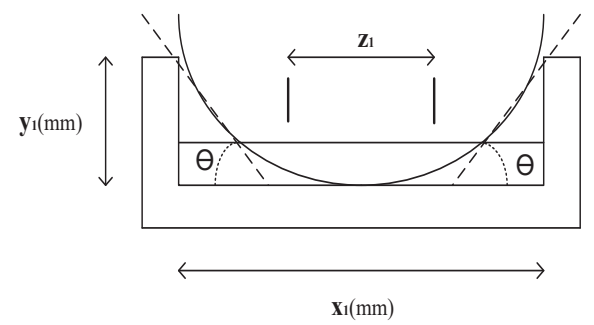

(a)

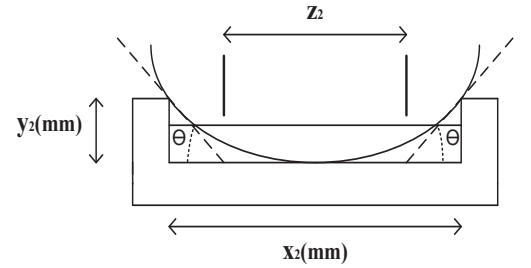

(b)

Fig. 3 (a). Diagram of side-etching using flat-base plate $80 \mathrm{~mm}$ based $\left(x_{1} \mathrm{~mm}\right) \times 25 \mathrm{~mm}$ height ( $\left.\mathrm{y}_{1} \mathrm{~mm}\right)$, Fig.3 (b). Diagram of side-etching using flat-base plate $60 \mathrm{~mm}$ based $\left(\mathrm{x}_{2} \mathrm{~mm}\right) \times 10$ $\mathrm{mm}$ height $\left(\mathrm{y}_{2} \mathrm{~mm}\right)$.

Fig. 3. (a). shows the diagram of the etching process using the flat base plate of size of $80 \mathrm{~mm}$ based $\times 25 \mathrm{~mm}$ height. The bending of the fiber influences the etching process since the fiber has to be held stress-free. If the fiber is under bending stress, once the fiber gets brittle, the fiber is easier to break into two as in Fig. 4. The angle $\Theta_{1}$ in Fig. 3. (a) is considerably big compared to $\Theta_{2}$ in Fig. x. (b) and the angle influences the bending applied onto the fiber. Fig. 3. (b) shows the diagram of the etching process using flat base plate of size $60 \mathrm{~mm}$ based $\times 10 \mathrm{~mm}$ height.

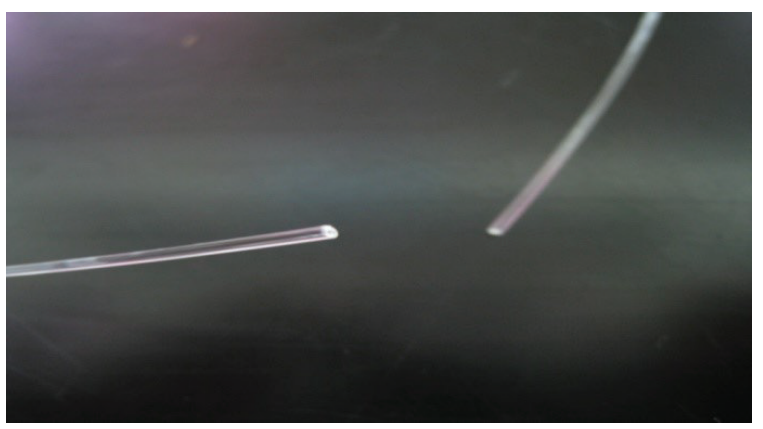

Fig. 4. Broken fiber due to brittleness and bending stress

Less bending stress is applied on the fiber and the fiber is held with small tension applied while the fiber is dipped in chemical solvent. Fig. 4. shows the fiber broke into two due to the factor of brittleness and bending stress applied onto the fiber in process of etching. 


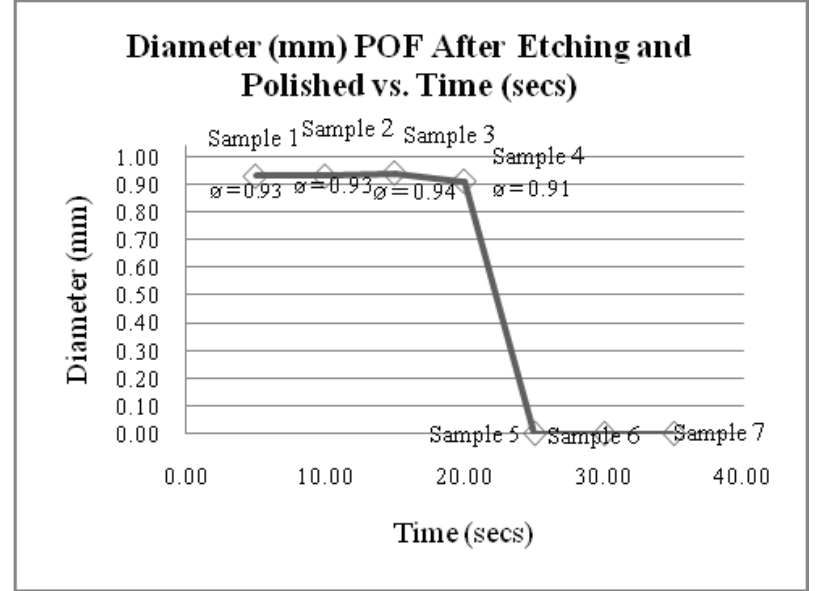

Fig. 5. Time (secs) vs Diameter ( $\mathrm{mm}$ ) for etched and polished POF samples using base-plate $80 \mathrm{~mm} \times 25 \mathrm{~mm}$.

Fig. 5. above is based on experiment done using flatbase plate of $80 \mathrm{~mm}$ based $\times 25 \mathrm{~mm}$ height. In addition to etching, for this particular experiment, polishing is done onto the surface of the affected region in order to ensure that the cladding layer is taken off entirely. However, it is crucial to notice that after 20 secs of dipping the fiber strand in the solvent, the samples broke. Analysis shows that it is due to the stress-fracture bending when the fiber region that exposed to the solvent gets brittle and resulted in breaking the fiber strand into two.

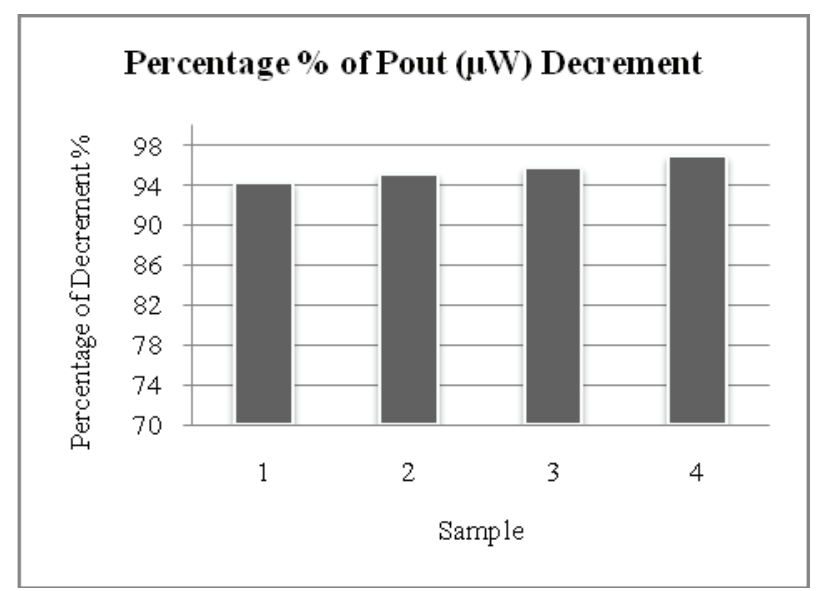

Fig. 6. Decrement in term of percentage of Output power $(\mu \mathrm{W})$ after samples are etched and polished.

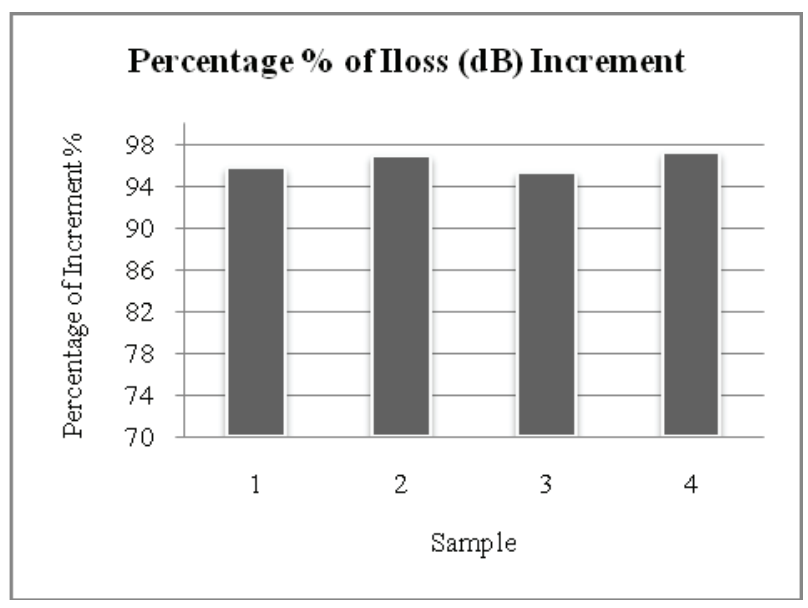

Fig. 7. Increment in term of percentage of Insertion loss (dB) after samples are etched and polished.

Fig. 6. and Fig. 7. show the after etching loss measurement for the four samples that didn't break. As the etching process gets longer i.e more than 5 secs, the output power decreased. When the light signal is transmitted through the fiber, the light spread out at the affected region and this might contribute to the decrement of the output power. Due to this behavior, the insertion loss increases.

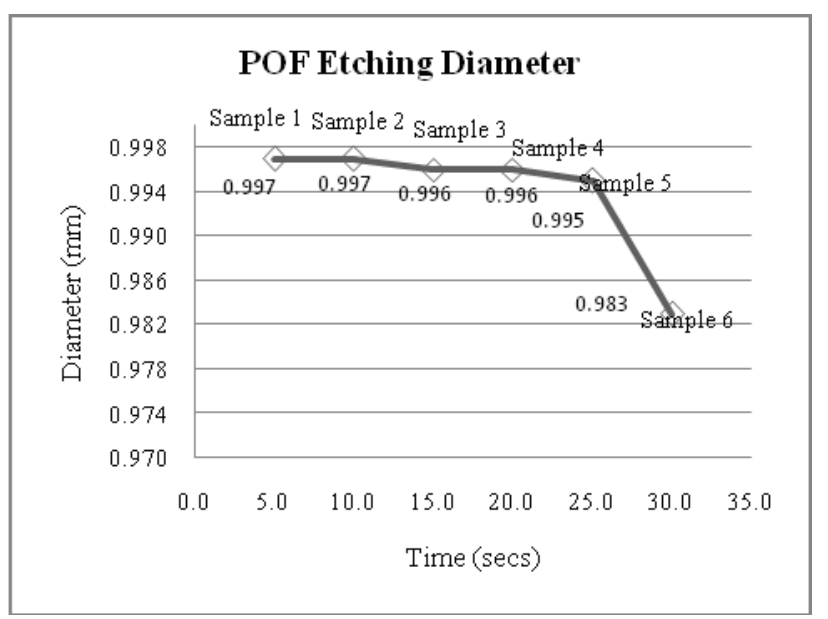

Fig. 8. Time (secs) vs Diameter ( $\mathrm{mm}$ ) for etched and unpolished region of POF samples using base-plate $60 \mathrm{~mm} \times$ $10 \mathrm{~mm}$.

For the etching process done using flat-base plate of 60 $\mathrm{mm}$ based $\times 10 \mathrm{~mm}$ height, six samples are successfully etched with longer time taken that is 30 secs as in Fig. 8. This experiment excludes the polishing of the etched area and let alone only the etching process to dissolve the cladding layer. The physical attribute of the plate contributed to the less bending of the fiber strand and less stress-fracture effect on the fiber as it gets brittle when dipped in the solvent. 


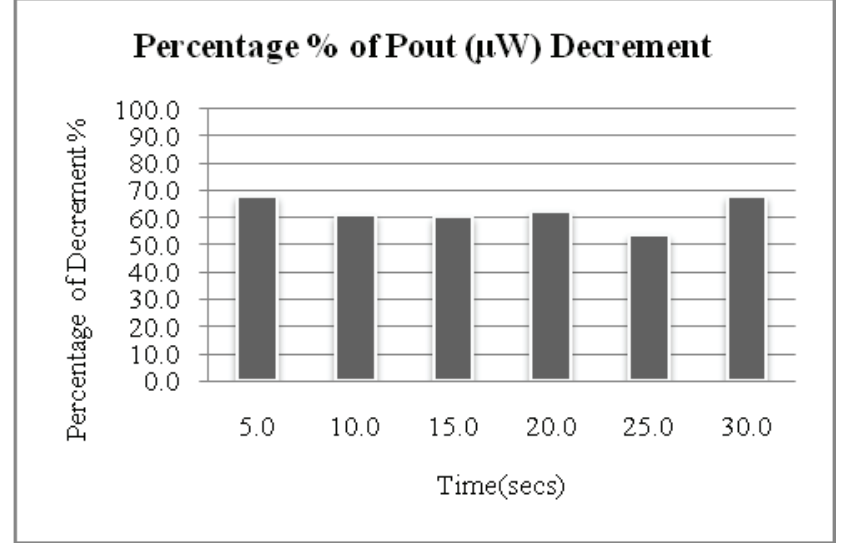

Fig. 9. Decrement in term of percentage of Output power $(\mu \mathrm{W})$ after samples are etched.

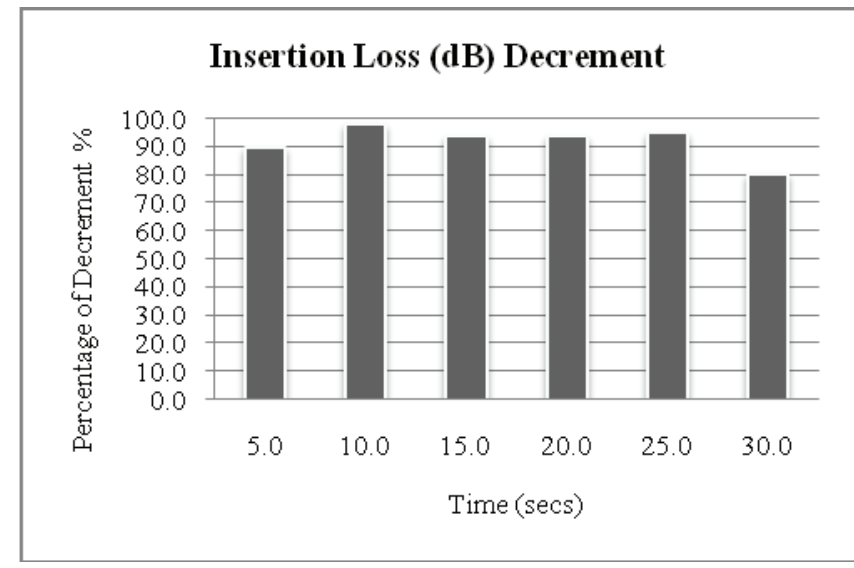

Fig. 10. Increment in term of percentage of Insertion loss (dB) after samples are etched.

Fig. 9. and Fig. 10. show the output power decrement and increment of the insertion loss measurement taken after the etching process. It can be seen that the output power decrement is not much differ from each other. Same behavior goes for insertion loss measurement taken. Since no polishing is applied onto the surface of the etched region, the behavior of the light transmission differs from the previous experiment. However, cloudy surface can be observed surrounded the etched region. This is due to the cladding stripping or etching is in progress, thus, unclear surface or cloudy surface is detected around the affected region. Longer time have to be applied during etching process to ensure that the cladding layer is fully taken off appropriately. This is proved by the diameter of the fiber after etching.

\section{Discussions}

The affected region that has been tapered using the solvent left only the pure core material section and the properties of the PMMA remains as flexible. The etching process produces through this method have the same optical properties like other multimode fiber tapers [4]. In large core multimode optical fiber such as POF, the number of guided modes can be approximated as explained in Senior [5],

$$
\mathrm{M}=\left(\mathrm{kr}_{\mathrm{c}} \mathrm{N}\right)^{2}=\left(\mathrm{kr}_{\mathrm{c}}\left(\mathrm{n}_{\mathrm{o}}^{2}-\mathrm{n}_{1}^{2}\right)^{1 / 2}\right)^{2}
$$

where $\mathrm{k}$ is the fiber propagation constant, $\mathrm{r}_{\mathrm{c}}$ is the radius of the core and $\mathrm{N}$ is the numerical aperture, $\mathrm{n}_{0}$ and $\mathrm{n}_{1}$ are the refractive indices of the core and cladding respectively. Losses of a coupler consist of the desired splitting loss and the undesired excess loss that exists beyond the splitting loss. Typical excess losses are on the order of $1 \mathrm{~dB}[6]$. The etching technique produces a tapered fiber that requires no alignment and has a constant attenuation of low-order modes. Modal redistribution length of the fiber is about few hundred meters thus the effect of tapers is local length [2]. If bare core is surrounded by an absorbing medium such as a liquid, the effective absorption coefficient of the evanescent field is inversely proportional to the radius [7].Thus, in this research, a coupler can be developed by aligning two bare core in close proximity to each other and to lessen the loss by the evanescent field effect, liquid such as glue having appropriate refractive index will be used surrounding the two fibers.

According to Huang [8], the simplest model for the coupled waveguide system is a directional coupler which consists of two uniform, parallel waveguides placed in close proximity. However, exact analytical solutions for practical directional coupler are difficult to find. Coupled mode theory gives simple analytical solutions that provide insight into the mode coupling process in directional coupler. When two guided modes are brought into close proximity, the modes will couple to each other as the results of evanescent fields.

\section{Conclusion}

The research presented explains the initial step in developing directional coupler using etching method. Apart from previous parameters analyzed, other parameters that are considered for future development are cost effective and environmental friendly fabrication, uniformity of the device, and excess loss. The applications of the fabricated device include vehicle network, sensor system, wavelength division multiplexing, smart housing network and video surveillance network for home security. The etching process done in realizing the coupler device is simple, low cost and requires only several equipment and it is a safe procedure since the solvent used doesn't involve harmful concentrated acids. Future research includes producing waist diameters less than $\varnothing=0.98$ to ensure optimum mode coupling. The measured parameters of the tapered POF offer significant advantages especially for performance of coupler that will be developed in the future. 


\section{References}

1. J. Crisp., Introduction to fiber optics (1996).

2. D.F.Merchant, P.J. Scully, N.F.Schmitt, Chemical tapering of polymer optical fibre, (Elsevier, 1998).

3. Understanding optical communication, (IBM Corporation, International Technical Support Organization, 1998).

4. A.W. Snyder, J.D.Love, Optical waveguide theory, (Chapman and Hall, 1983).

5. J.M.Senior, Optical fibre communication principles and practice, (Prentice-Hall, London, 1985).

6. J. Powers, An introduction to fiber optic systems, $2^{\text {nd }}$ ed., (MacGraw-Hill, 1999).

7. V.Ruddy, Fiber and Integrated Optics, 9(2), 143-151 (1990).

8. W.P.Huang, Optical Society of America, 11 (3) (1994). 